Article

\title{
Genome-Wide Identification of QTLs for Grain Protein Content Based on Genotyping-by-Resequencing and Verification of qGPC1-1 in Rice
}

\author{
Yi-Bo Wu ${ }^{1,+}{ }^{,}$Guan Li ${ }^{1,+}$, Yu-Jun Zhu ${ }^{1}$, Yi-Chen Cheng ${ }^{1}$, Jin-Yu Yang ${ }^{1}$, Hui-Zhe Chen ${ }^{1}$, \\ Xian-Jun Song ${ }^{2}$ and Jie-Zheng Ying ${ }^{1, *(\mathbb{D})}$ \\ 1 State Key Laboratory of Rice Biology and Chinese National Center of Rice Improvement, China National \\ Rice Research Institute, Hangzhou 310006, China; 15978677129@163.com (Y.-B.W.); li@ricescience.org (G.L.); \\ yjzhu2013@163.com (Y.-J.Z.); 13872209530@163.com (Y.-C.C.); yang_15271211512@163.com (J.-Y.Y.); \\ chenhuizhe@163.com (H.-Z.C.) \\ 2 Key Laboratory of Plant Molecular Physiology, Institute of Botany, the Chinese Academy of Sciences, \\ Beijing 100093, China; songxj@ibcas.ac.cn \\ * Correspondence: yingjiezheng@caas.cn; Tel.: +86-571-63370364 \\ + These authors contributed equally to this study.
}

Received: 22 November 2019; Accepted: 7 January 2020; Published: 9 January 2020

check for updates

\begin{abstract}
To clarify the genetic mechanism underlying grain protein content (GPC) and to improve rice grain qualities, the mapping and cloning of quantitative trait loci (QTLs) controlling the natural variation of GPC are very important. Based on genotyping-by-resequencing, a total of 14 QTLs were detected with the Huanghuazhan/Jizi1560 (HHZ/JZ1560) recombinant inbred line (RIL) population in 2016 and 2017. Seven of the fourteen QTLs were repeatedly identified across two years. Using three residual heterozygote-derived populations, a stably inherited QTL named as qGPC1-1 was validated and delimited to a $\sim 862 \mathrm{~kb}$ marker interval JD1006-JD1075 on the short arm of chromosome 1 . Comparing the GPC values of the RIL population determined by near infrared reflectance spectroscopy (NIRS) and Kjeldahl nitrogen determination (KND) methods, high correlation coefficients ( 0.966 and 0.983) were observed in 2016 and 2017. Furthermore, 12 of the 14 QTLs were identically identified with the GPC measured by the two methods. These results indicated that instead of the traditional KND method, the rapid and easy-to-operate NIRS was suitable for analyzing a massive number of samples in mapping and cloning QTLs for GPC. Using the gel-based low-density map consisted of 208 simple sequence repeat (SSR) and insert/deletion (InDel) markers, the same number of QTLs (fourteen) were identified in the same HHZ/JZ1560 RIL population, and three QTLs were repeatedly detected across two years. More stably expressed QTLs were identified based on the genome resequencing, which might be attributed to the high-density map, increasing the detection power of minor QTLs. Our results are helpful in dissecting the genetic basis of GPC and improving rice grain qualities through molecular assisted selection.
\end{abstract}

Keywords: quantitative trait locus; grain protein content; single nucleotide polymorphism; residual heterozygote; rice (Oryza sativa); specific length amplified fragment sequencing; Kjeldahl nitrogen determination; near infrared reflectance spectroscopy

\section{Introduction}

Rice grain quality, including appearance, milling, cooking and eating, as well as nutritional qualities, determines the market value, and is getting more and more concern from rice researchers, 
producers and consumers [1,2]. Grain protein content (GPC) is not only one key factor determining nutritional quality, but is also closely associated with cooking and eating qualities [3,4]. Generally, the increase of GPC may consequently lead to low eating quality.

Compared to the protein content of other cereal crops such as wheat and barley, GPC in rice is relatively low, with a mean about $8.0 \%$ and a range of $4.9 \%$ to $19.3 \%$ in the indica subspecies and $5.9 \%$ to $16.5 \%$ in the japonica subspecies [5]. As a typical quantitative trait, GPC in rice is easily affected by environmental conditions, especially the level of nitrogen fertilizer in the field, which makes it very difficult to manipulate in a traditional breeding program. Therefore, illuminating the genetic basis of GPC makes a lot of sense in constructing a molecular marker-assisted selection system to improve rice grain quality [6,7].

Quantitative trait locus (QTL) analysis is the main strategy for dissecting the genetic mechanism underlying a target quantitative trait. During the past two decades, hundreds of QTLs for GPC in rice were detected throughout the entire 12 chromosomes, using different mapping populations, including the recombinant inbred line (RIL) [3,8-13], double haploid population [14-17], chromosome segment substitution line $[4,18,19]$ and the backcross-inbred population [20]. As GPC is sensible to environmental factors, QTLs controlling the GPC are difficult to be repeatedly identified in different populations, or in the same population under different environments [19]. Till now, only two QTLs, $q P C 1$ and $q G P C-10$, have been map-based cloned and functionally analyzed. $q P C 1$ was found on the long arm of chromosome 1, which encodes a putative amino acid transporter OsAAP6 and functions as a positive regulator of GPC in rice [6]. $q$ GPC-10 located on chromosome 10 encodes a glutelin type-A2 precursor, and is also a positive regulator of GPC [7]. Besides, another stably inherited QTL $q P C-1$ that is nonallelic to $q P C 1$ was validated and delimited to a $41-\mathrm{kb}$ region on the long arm of chromosome 1 [19]. Owing to the detection instability of GPC QTLs, it is important to confirm the genetic effect of the QTLs detected in the primary mapping before their map-based cloning and application in the improvement of rice nutritional quality.

Genotyping and phenotyping of the mapping population are two essential components for QTL analysis. With the development of next-generation sequencing, genotyping-by-sequencing becomes a feasible technique to rapidly identify a huge number of single nucleotide polymorphisms (SNPs) throughout the whole genome. Then, a high-density linkage map can be constructed with saturated SNP markers, while most current maps are low-density, and only contain hundreds of gel-based DNA markers, such as restriction fragment length polymorphism and simple sequence repeat (SSR) markers. The detection power of minor QTLs can be improved, and the confidence interval of the QTL can be reduced in a high-resolution linkage map [21]. Among the most common genotyping-by-sequencing methods, specific length amplified fragment sequencing (SLAF-seq) is acceptable as an efficient and high-resolution technology with a relatively lower sequencing cost [22]. With the availability of rice genome draft, genotyping-by-resequencing (GBR) has been applied in linkage mapping and genome-associated analysis to map QTLs for important agronomic traits. Nevertheless, for GPC, the information of QTL identified by GBR of a bi-parent population is still limited in rice.

GPC is traditionally measured by the Kjeldahl nitrogen determination (KND), which is time-consuming and needs a large amount of chemicals such as strong acid and alkali. Therefore, this KND method is difficult for the measurement of a massive number of samples, which is usually necessary in the map-based cloning of a target QTL. Compared to the KND method, near infrared reflectance spectroscopy (NIRS) is a promising technique that is fast and easy-to-use [23]. A lot of QTLs were identified, and two major QTLs have been successfully isolated through the NIRS method. However, it keeps unknown whether there is difference in the detection power of QTLs for GPC between the two methods.

In this study, we mainly completed the following research objectives. First, we analyzed the correlation between the GPC values measured by the KND and NIRS methods, and validated the feasibility of mapping QTLs for GPC using NIRS instead of KND. Second, we identified QTLs for GPC with a high-resolution genetic map containing 18,194 SNP markers in an RIL population, which was 
derived from a cross between an indica variety Huanghuazhan (HHZ) and a japonica accession Jizi1560 (JZ1560). Third, we compared the GPC QTLs identified with the high- and low-density map in the same HHZ/JZ1560 RIL population. Finally, one stably inherited major QTL (qGPC1-1) located on the short arm of chromosome 1 was validated using three secondary populations developed from three residual heterozygotes $(\mathrm{RH})$ with the heterozygous genotype at the target interval.

\section{Results}

\subsection{Phenotypic Variation and Correlation Analysis}

In 2016 and 2017, the GPC of the HHZ/JZ1560 RIL population and the parents was measured by NIRS and KND, respectively. The descriptive statistics of phenotypic variation are shown in Table 1. Significant phenotypic differences were observed between the parents, and the GPC of JZ1560 was higher than that of HHZ in both years. Frequency distributions of GPC in the brown rice flour of RILs and the parents were plotted (Figure 1). The GPC of the RILs showed similar distribution either measured by the KND or NIRS method in each year. Phenotypic variation was continuously distributed with low skewness and kurtosis, showing a typical pattern of quantitative variation. Using the KND method, the GPC of the RILs showed a wide variation from $7.27 \%$ to $15.78 \%$ in 2016 and from $7.39 \%$ to $15.98 \%$ in 2017 (Figure 1). Continuous segregation and significant transgressive segregation at two directions suggested polygenic control underlying this trait.

Table 1. Descriptive statistics of the grain protein content (GPC, \%) in the recombinant inbred line (RIL) population and the parents in the two years.

\begin{tabular}{cccccccccc}
\hline \multirow{2}{*}{ Year } & \multirow{2}{*}{ Method } & \multicolumn{4}{c}{ RIL Population $(\boldsymbol{n}=\mathbf{2 8 0})$} & \multicolumn{3}{c}{ Parental Mean $(\boldsymbol{n}=\mathbf{1 6})$} & \multirow{2}{*}{$\boldsymbol{p}$} \\
\cline { 3 - 9 } & & Mean \pm SD & CV & Range & Skewness & Kurtosis & HHZ & JZ1560 & \\
\hline \multirow{2}{*}{2016} & NIRS & $12.44 \pm 1.59$ & 0.13 & $8.35-16.35$ & 0.20 & -0.26 & $10.14 \pm 0.02$ & $13.16 \pm 0.01$ & $<0.001$ \\
& KND & $11.68 \pm 1.62$ & 0.14 & $7.27-15.78$ & 0.18 & -0.30 & $9.39 \pm 0.11$ & $13.06 \pm 0.33$ & \\
2017 & NIRS & $10.67 \pm 1.40$ & 0.13 & $7.29-15.90$ & 0.28 & 0.06 & $8.44 \pm 0.03$ & $10.92 \pm 0.01$ & $<0.001$ \\
& KND & $10.64 \pm 1.39$ & 0.13 & $7.39-15.98$ & 0.29 & 0.15 & $8.54 \pm 0.00$ & $10.62 \pm 0.36$ & \\
\hline
\end{tabular}

NIRS: near infrared reflectance spectroscopy; KND: Kjeldahl nitrogen determination; $p$ : two-tailed $p$ value of student's $t$ test.
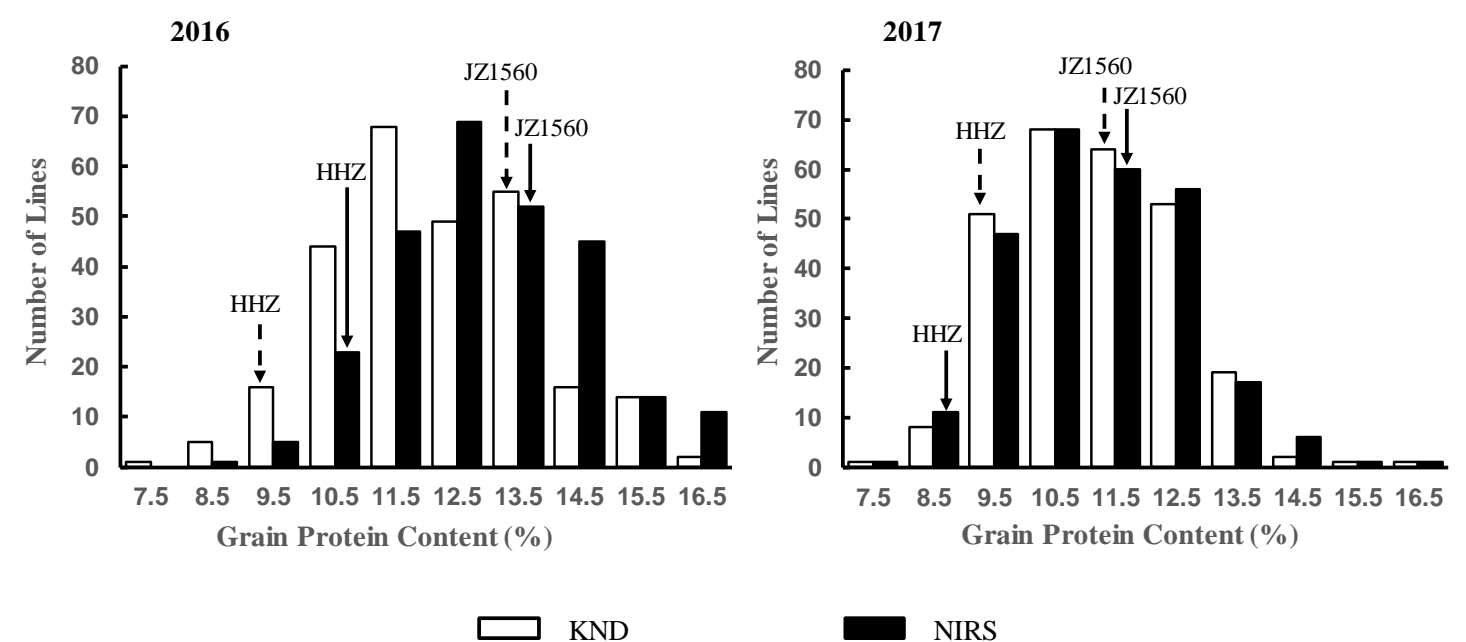

Figure 1. Frequency distributions of grain protein content (GPC) measured by near infrared reflectance spectroscopy (NIRS) and Kjeldahl nitrogen determination (KND) methods in the recombinant inbred line (RIL) population. Parental values are indicated by arrows.

Significant positive correlations between the GPC values measured by the two methods or in different years were found in the RIL population (Table 2). However, correlation coefficients for GPC determined by KND and NIRS methods as 0.966 in 2016 and 0.983 in 2017 were much higher than that 
of GPC measured with the same method in different years as 0.638 for NIRS and 0.631 for KND. This implied that GPC determined by NIRS was quite consistent with that by the KND method, and GPC was strongly influenced by environmental factors.

Table 2. Correlation coefficients of GPC between two methods and between different years from 280 RILs.

\begin{tabular}{llll}
\hline & 16NIRS & 16KND & 17NIRS \\
\hline 16KND & $0.966^{* *}$ & & \\
17NIRS & $0.638^{* *}$ & $0.651^{* *}$ & \\
17KND & $0.614^{* *}$ & $0.631^{* *}$ & $0.983^{* *}$ \\
\hline
\end{tabular}

16NIRS, 17NIRS: GPC of 280 RILs in 2016 and 2017 measured by near infrared reflectance spectroscopy (NIRS); 16KND, 17KND: GPC of 280 RILs in 2016 and 2017 measured by Kjeldahl nitrogen determination (KND). ** highly significant correlations at the 0.01 level.

\subsection{QTL Analysis of Grain Protein Content in the HHZ/JZ1560 RIL Population}

Combining the high-density genetic map containing 18,194 SNP markers with the GPC means of each RIL, 14 QTLs were detected on the whole genome except for chromosomes 6, 9 and 12 with each QTL explaining $0.81 \%-18.59 \%$ of the phenotypic variations (Table 3, Figures 2 and 3). Among the QTLs, 12 were identified in 2016 and nine in 2017. The detailed description of each QTL including peak location, peak LOD value, additive effect and percentage of total phenotypic variations $\left(R^{2}\right)$ are showed in Table 3. Except for $q G P C 2, q G P C 8$ and $q G P C 10$, the enhancing alleles for GPC were derived from JZ1560 at the remaining 11 loci as the brown rice of JZ1560 contained significantly higher GPC (Table 1).

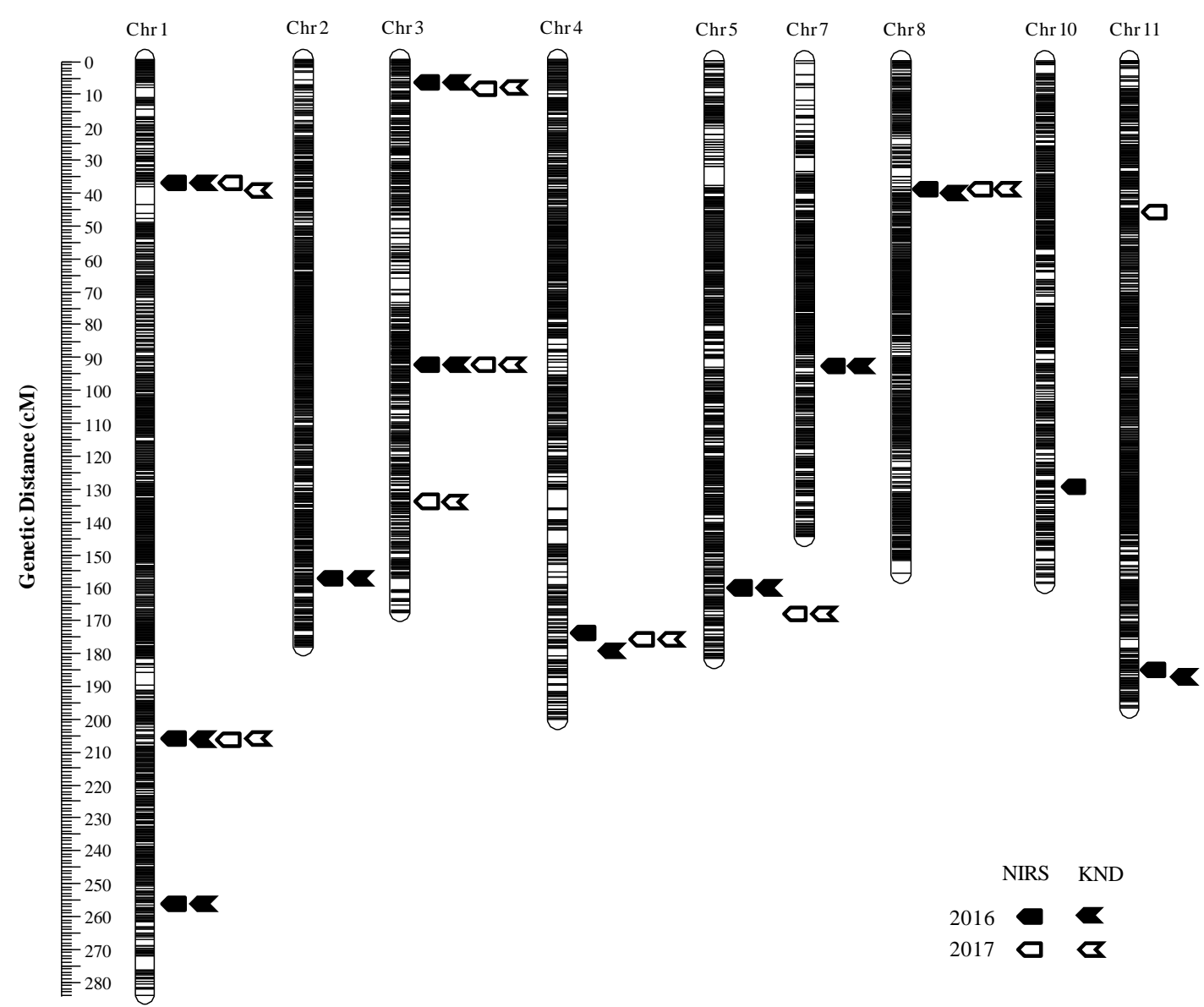

Figure 2. High-density linkage map based on genotyping-by-resequencing showing the most likely positions of QTLs for GPC measured by NIRS and KND methods in the HHZ/JZ1560 RIL population. 
Table 3. QTLs for GPC based on genotyping-by-resequencing in the RIL population.

\begin{tabular}{|c|c|c|c|c|c|c|c|}
\hline QTL & Year & Method & Marker Interval & Position (cM) & LOD & $A$ & $R^{2}(\%)$ \\
\hline \multirow{4}{*}{$q G P C 1-1$} & 2016 & NIRS & Marker37041-37509 & 36.46 & 7.96 & 0.57 & 9.14 \\
\hline & 2016 & KND & Marker37041-37509 & 36.46 & 9.72 & 0.55 & 10.89 \\
\hline & 2017 & NIRS & Marker37041-37509 & 36.46 & 9.59 & 0.58 & 11.85 \\
\hline & 2017 & KND & Marker37508 & 38.74 & 8.72 & 0.47 & 11.05 \\
\hline \multirow[t]{4}{*}{$q G P C 1-2$} & 2016 & NIRS & Marker138502 & 205.52 & 2.12 & 0.45 & 5.53 \\
\hline & 2016 & KND & Marker138502-136654 & 205.52 & 2.44 & 0.38 & 5.20 \\
\hline & 2017 & NIRS & Marker136541-138500 & 205.88 & 3.07 & 0.41 & 5.88 \\
\hline & 2017 & KND & Marker138502-139278 & 205.52 & 3.06 & 0.36 & 6.41 \\
\hline \multirow[t]{2}{*}{$q G P C 1-3$} & 2016 & NIRS & Marker208637-210208 & 255.84 & 3.00 & 0.21 & 1.18 \\
\hline & 2016 & KND & Marker207139-209159 & 255.84 & 2.62 & 0.15 & 0.81 \\
\hline \multirow[t]{2}{*}{$q G P C 2$} & 2016 & NIRS & Marker413084-424309 & 156.64 & 3.96 & -0.36 & 3.68 \\
\hline & 2016 & KND & Marker413084-424409 & 156.64 & 3.91 & -0.31 & 3.47 \\
\hline \multirow[t]{4}{*}{ qGPC3-1 } & 2016 & NIRS & Marker449522-449025 & 5.95 & 11.15 & 0.78 & 16.86 \\
\hline & 2016 & KND & Marker449522-449025 & 5.95 & 12.68 & 0.72 & 18.59 \\
\hline & 2017 & NIRS & Marker450509-451398 & 7.80 & 6.34 & 0.46 & 7.21 \\
\hline & 2017 & KND & Marker450216-451025 & 7.61 & 6.77 & 0.41 & 8.40 \\
\hline \multirow[t]{4}{*}{ qGPC3-2 } & 2016 & NIRS & Marker567334-573384 & 91.73 & 2.41 & 0.30 & 2.50 \\
\hline & 2016 & KND & Marker571649-571070 & 91.73 & 2.52 & 0.26 & 2.53 \\
\hline & 2017 & NIRS & Marker571649-572521 & 91.73 & 2.31 & 0.36 & 4.63 \\
\hline & 2017 & KND & Marker571649-573384 & 91.73 & 2.38 & 0.30 & 4.53 \\
\hline \multirow[t]{2}{*}{ qGPC3-3 } & 2017 & NIRS & Marker620694-621697 & 133.44 & 5.74 & 0.39 & 5.44 \\
\hline & 2017 & KND & Marker620694-621697 & 133.44 & 6.11 & 0.36 & 6.29 \\
\hline \multirow[t]{4}{*}{$q G P C 4$} & 2016 & NIRS & Marker779713-795626 & 173.67 & 3.90 & 0.45 & 5.61 \\
\hline & 2016 & KND & Marker790607-796001 & 178.83 & 4.58 & 0.39 & 5.47 \\
\hline & 2017 & NIRS & Marker792925-793575 & 175.36 & 2.93 & 0.35 & 4.26 \\
\hline & 2017 & KND & Marker792925-796001 & 175.36 & 3.56 & 0.31 & 4.89 \\
\hline \multirow{4}{*}{$q G P C 5$} & 2016 & NIRS & Marker940773-956686 & 159.63 & 4.09 & 0.35 & 3.47 \\
\hline & 2016 & KND & Marker951188-956259 & 159.63 & 3.19 & 0.24 & 2.12 \\
\hline & 2017 & NIRS & Marker942375-968984 & 167.68 & 5.02 & 0.41 & 6.09 \\
\hline & 2017 & KND & Marker941389-968984 & 167.68 & 4.61 & 0.32 & 5.21 \\
\hline \multirow[t]{2}{*}{$q G P C 7$} & 2016 & NIRS & Marker1254363-1255283 & 92.11 & 3.46 & 0.50 & 6.84 \\
\hline & 2016 & KND & Marker1254363-1255919 & 92.11 & 3.83 & 0.44 & 7.15 \\
\hline \multirow[t]{4}{*}{$q G P C 8$} & 2016 & NIRS & Marker1330696-1330654 & 38.51 & 7.81 & -0.57 & 9.23 \\
\hline & 2016 & KND & Marker1330714-1331214 & 39.43 & 7.10 & -0.46 & 7.71 \\
\hline & 2017 & NIRS & Marker1330696-1330654 & 38.51 & 2.12 & -0.28 & 2.70 \\
\hline & 2017 & KND & Marker1330696 & 38.33 & 2.55 & -0.25 & 3.20 \\
\hline qGPC10 & 2016 & NIRS & Marker1714254-1714662 & 129.12 & 2.84 & -0.43 & 5.18 \\
\hline qGPC11-1 & 2017 & NIRS & Marker1789482-1788848 & 45.47 & 2.08 & 0.33 & 3.89 \\
\hline \multirow[t]{2}{*}{ qGPC11-2 } & 2016 & NIRS & Marker1901374-1901256 & 184.63 & 2.03 & 0.46 & 5.84 \\
\hline & 2016 & KND & Marker1902918-1902895 & 186.73 & 2.06 & 0.36 & 4.66 \\
\hline
\end{tabular}

NIRS: near infrared reflectance spectroscopy; KND: Kjeldahl nitrogen determination; $A$ : additive effect of replacing a maternal allele with a paternal allele; $R^{2}$ : proportion of the phenotypic variance explained by the QTL. 


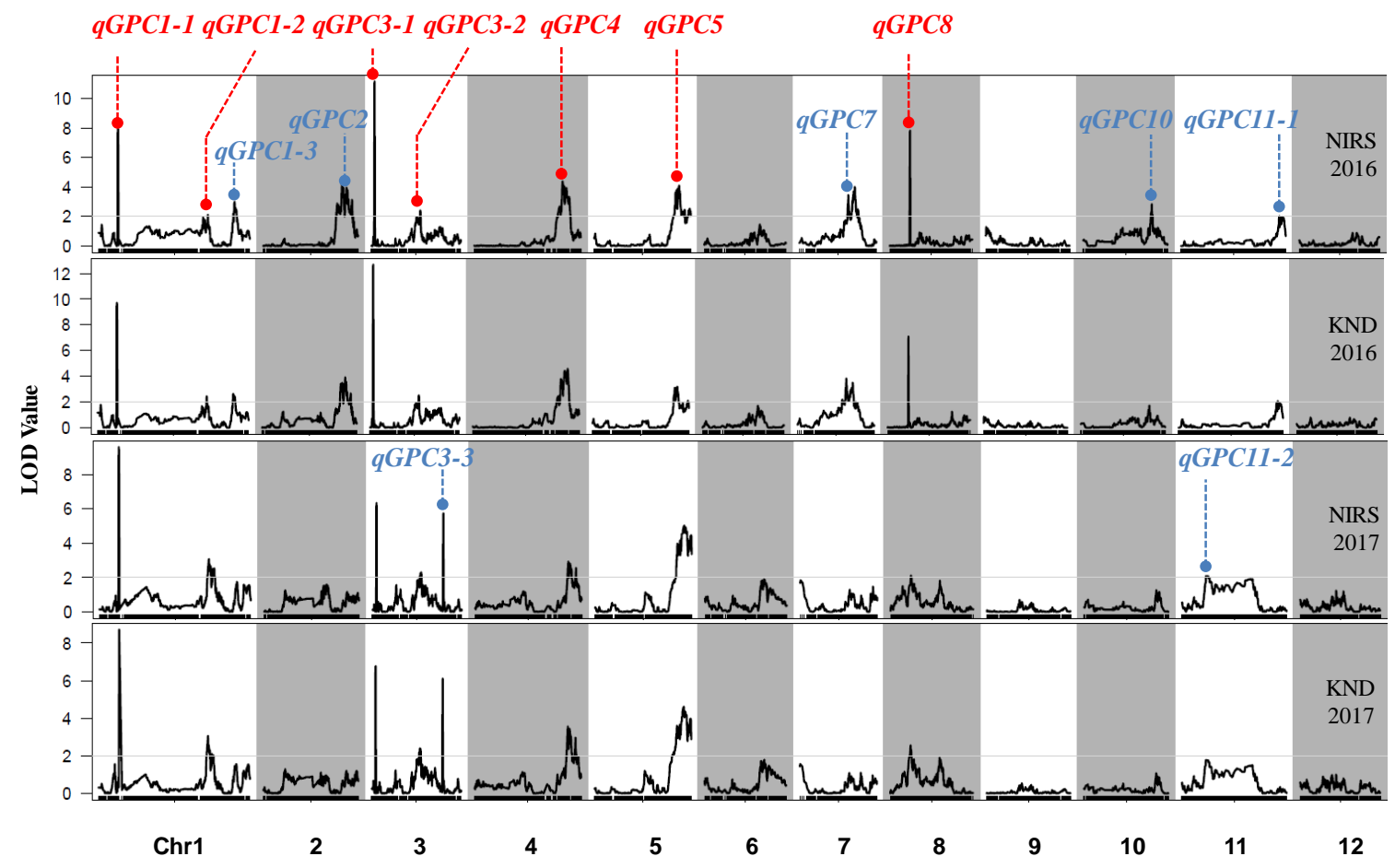

Figure 3. The identified QTLs for GPC measured by the NIRS and KND methods through analyzing the SNP genotypes and corresponding phenotypes of the 280 RILs. Red font indicates that QTLs were detected in both 2016 and 2017, and blue font indicates that QTLs were identified in either year 2016 or 2017.

In order to find the difference in the detection power of QTLs using different measurement methods of GPC, we further compared the QTLs for GPC determined by the NIRS and KND methods. In 2016, we identified 12 QTLs for GPC measured by NIRS and 11 QTLs for GPC measured by KND, which explained $55.07 \%$ and $48.25 \%$ of the total phenotypic variations, respectively. Eleven QTLs were commonly mapped using the two measurement methods, and one QTL ( $q$ GPC10) with a small genetic effect was only detected using the NIRS method (Figures 2 and 3, Table 3). Similar results were observed in 2017. Eight common QTLs were identified by both the measurement methods, and only one minor QTL ( $q$ GPC11) was mapped on chromosome 11 using the NIRS method. This indicated that QTLs for GPC were coincided between the two GPC measurement methods. Seven of the fourteen QTLs, qGPC1-1, qGPC1-2, qGPC3-1, qGPC3-2, qGPC4, qGPC5 and $q G P C 8$, were repeatedly identified in both years. The remaining seven QTLs were only detected in one year.

QTL analysis was also performed using a low-density genetic map containing 208 SSR and InDel markers and a total of 14 QTLs were detected in the same HHZ/JZ1560 RIL population (Figure 4, Table S1). Compared with the seven stably inherited QTLs identified in the high-density genetic map, only three QTLs including $q G P C 1, q G P C 3-1$ and $q G P C 5$ were mapped at the same region and showed the similar effects for the two years using the low-density genetic map, suggesting that the high-density genetic map increased the detection power of QTLs for GPC. 

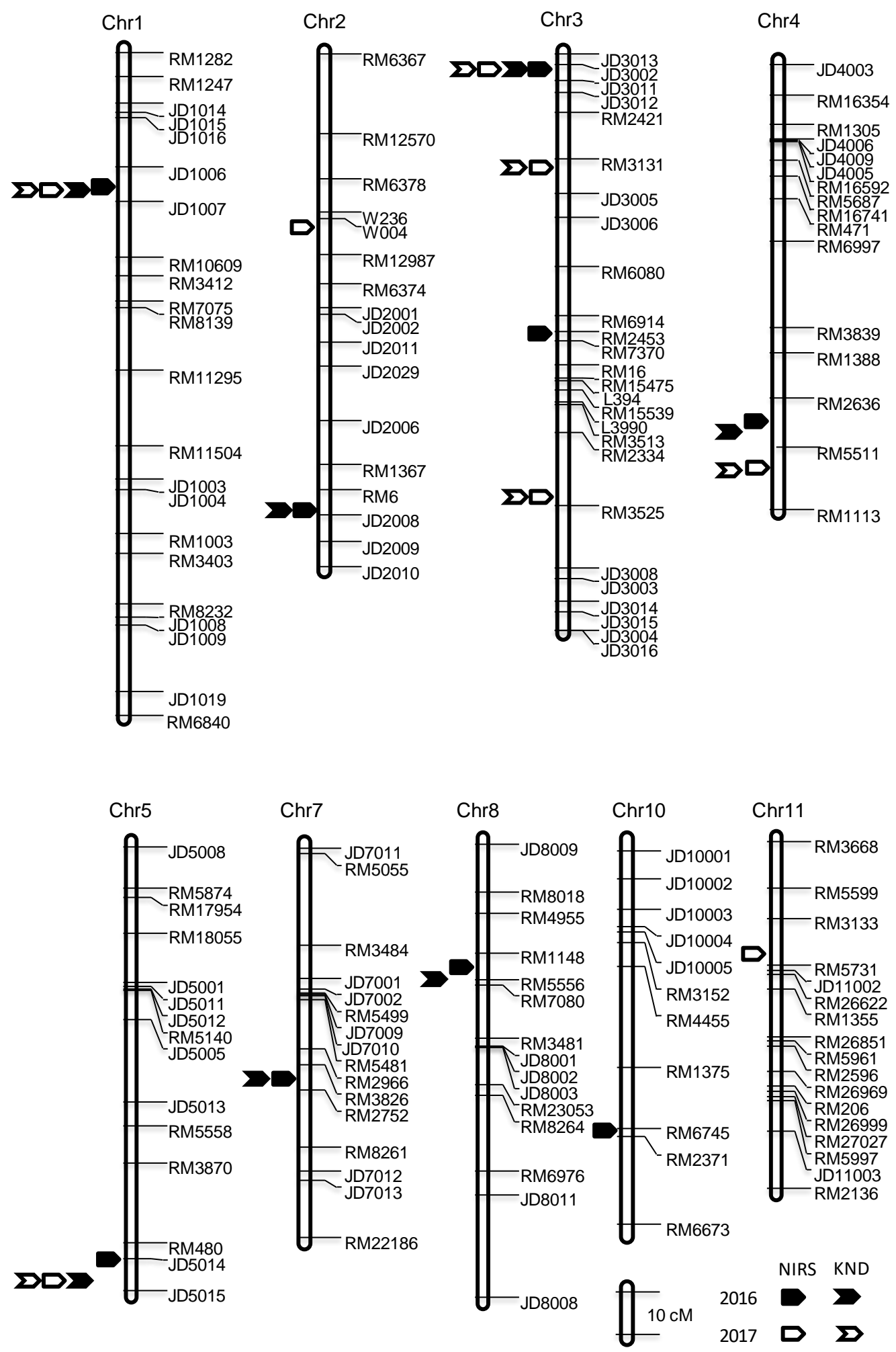

Figure 4. Low-density linkage map containing 208 gel-based SSR and InDel markers showing the most likely positions of QTLs for GPC measured by NIRS and KND methods in the HHZ/JZ1560 RIL population.

The $q$ GPC1-1 was detected on the short arm of chromosome 1 across two years using the high-density genetic map and accounted for $9.14 \%$ to $11.85 \%$ of the phenotypic variations. The allele from JZ1560 at this locus increased GPC by $0.47 \%-0.58 \%$. Corresponding to $q G P C 1-1, q G P C 1$ was mapped at the same location with the flanking markers JD1006 and JD1007 using the low-density genetic map in both years 
(Figure 4, Table S1). The $q$ GPC1 identified in this study contributed $11.78 \%$ to $13.33 \%$ of phenotypic variations with a relatively large additive effect ranging from $0.54 \%$ to $0.71 \%$ (Table S1).

\subsection{Validation and Delimitation of $q$ GPC1-1 Using RH-derived $F_{2}$ Populations}

To confirm the genetic effect and location of $q$ GPC1-1, three RH individuals were selected from one $\mathrm{F}_{8}$ RIL line with the heterozygous genotype covering the target marker interval of JD1006-JD1007. Three RH-derived $F_{2}$ populations, named as WB01, WB02 and WB03, were developed from the three plants with sequential heterozygous segments extending from JD1006 to JD1007, respectively. Based on the sequence differences between the parents HHZ and JZ1560 identified by 30-fold whole genome re-sequencing, additional six InDel markers were developed and used to genotype the three populations (Table S2). GPC was continuously distributed and ranged from $8.33 \%$ to $11.90 \%, 8.27 \%$ to $12.19 \%$ and $8.32 \%$ to $10.47 \%$ in WB01, WB02 and WB03 populations, respectively (Figure S1).

Three segmental linkage maps were constructed for WB01, WB02 and WB03, respectively (Figure 5). Combined the genotype and phenotype information, $q$ GPC1-1 was identified in WB01 and WB02 populations, with the JZ1560 allele always increasing GPC (Table 4; Figure 5). This QTL explained $26.00 \%$ and $27.40 \%$ of phenotypic variations with the similar additive effects of $0.36 \%$ and $0.39 \%$ in WB01 and WB02, respectively. No QTL for the GPC was detected in the WB03 population. Therefore, qGPC1-1 should be located within the common segregating regions of WB01 and WB02, but outside the segregating region of WB03. As shown in Figure 5, qGPC1-1 was delimited to the interval between JD1006 and JD1075 ( 862 kb) with a common segregating region JD1068-JD1075 and one flanking cross-over region JD1006-JD1068.

Table 4. QTLs for the GPC detected in the three residual heterozygote-derived $F_{2}$ populations.

\begin{tabular}{ccccccc}
\hline Population Name & Segregating Region & Sample & LOD & $\boldsymbol{A}$ & $\boldsymbol{D}$ & $\boldsymbol{R}^{\mathbf{2}} \mathbf{( \% )}$ \\
\hline WB01 & JD1006-JD1078 & 180 & 7.59 & 0.36 & 0.11 & 26.00 \\
WB02 & JD1068-JD1007 & 137 & 6.25 & 0.39 & 0.04 & 27.40 \\
WB03 & JD1075-JD1007 & 115 & ns & ns & ns & ns \\
\hline
\end{tabular}

$A$ : additive effect of replacing a maternal allele with a paternal allele; $D$ : dominance effect; $R^{2}$ : proportion of the phenotypic variance explained by the QTL; ns: no significance.

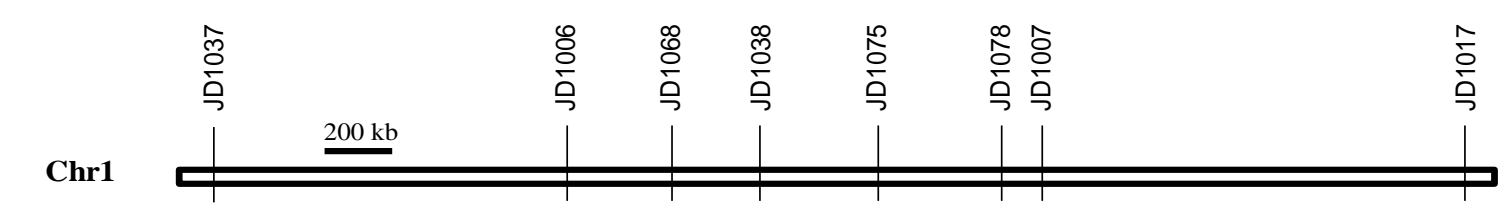

Population

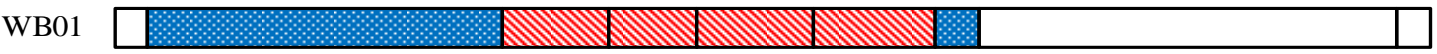

WB02

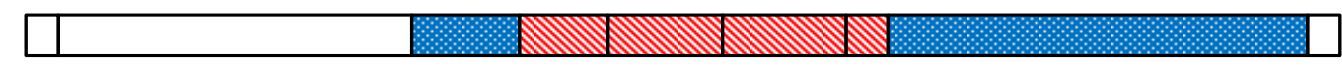

WB03

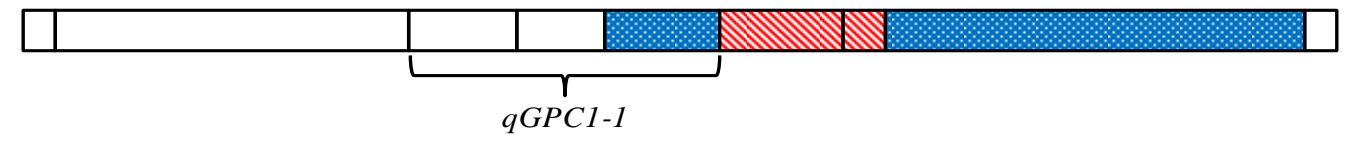

Homozygous Region $\mathbb{N}$ Segregating Region $\quad$ Cross-over Region

Figure 5. Genotypic compositions of the three residual heterozygote-derived $F_{2}$ populations in the segregating regions. 


\section{Discussion}

Elucidating the genetic mechanism of GPC accumulation is very important for regulating rice grain qualities in breeding. In the present study, we characterized the genetic basis of GPC and identified a total of 14 QTLs using the high-resolution map in the HHZ/JZ1560 RIL population. Although GPC is sensitive to environmental conditions and the QTLs for GPC are difficult to be repeatedly identified in different environments, the majority of the 14 QTLs have been reported in the previous studies. On the long arm of chromosome 1, QTLs for GPC have been reported in some studies including pro1 between RM226 and RM297 [15], $q P C-1$ between R888 and R1485 [13], $q P C 1$ between RM472 and RM104 [6], $q P C-1$ between RM1196 and RM302 [19] and TGP1b between RM1297 and RM1067 [4]. The qGPC1-2 and $q$ GPC1-3 were located in the adjacent chromosome regions with these reported QTLs, and the indica variety HHZ allele decreased the GPC. Two minor QTLs, qGPC2 and qGPC11-1, were also mapped at the similar locations with pro2 between RM6 and RM112 and pro11 between RM209 and RM229 [15]. On the chromosome 3, we detected three QTLs including $q G P C 3-1, q G P C 3-2$ and $q G P C 3-3$, and the enhancing alleles were all from the japonica rice JZ1560. The qGPC3-1 with the largest effect in 2016 and $q G P C 3-2$ were repeatedly detected as $q P C-3.1$ in the interval XNpb212-G1318 and $q P C-3.2$ in the interval R758-XNpb15, respectively [13]. The qGPC3-3 was located in the overlapping confidence interval with the QTLs for protein content in several previous reports $[13,14,18]$. These results indicated that there are multiple genetic factors controlling GPC on chromosome 3. The qGPC4 was detected within the $q P C-4$ region between RG214 and RG620 [12]. We still noted that $q G P C 5$ was repeatedly identified as qRPC5 for rice protein content in the interval RG435-RG172a using a doubled haploid population [16]. Although $q G P C 7$ was only detected in 2016, four QTLs were located in the same or adjacent regions as reported by previous studies $[3,4,8,17]$. On the chromosome $8, q G P C 8$ showed overlapping intervals with $c p 8.1, q P C-8 a$ and TGP8, which have been identified using different populations in different environments $[4,18,24]$. The $q P C 11-2$ with minor effect was mapped near to $q P C 11$ between RM202 and RM206 [25]. No QTL for GPC has been mapped in the region of qGPC10 on chromosome 10 before, therefore $q G P C 10$ might be newly detected in this study. Unlike many previous studies, we did not detect the QTL for GPC near to the $W x$ locus on chromosome $6[4,8,12,25]$. Over all, the locations of QTLs for GPC showed a significant similarity between our studies and the previous findings.

Of the 14 QTLs identified in this study, the $q G P C 1-1$ was a stably expressed QTL with a relatively large effect and it was repeatedly detected in both years. This QTL was also identified in the similar chromosome region in different populations and environments, implying that $q G P C 1-1$ plays an important role in controlling GPC $[4,11,18]$. Based on the primary mapping result, $q$ GPC1-1 was further validated and delimited in the interval JD1006-JD1075, corresponding to the 6.0-6.8 Mb region on the short arm of chromosome 1 in the Nipponbare genome [26]. The japonica rice JZ1560 allele contributed to the increase of GPC in the RH-derived $\mathrm{F}_{2}$ population (Table 4). Dissecting the genetic mechanism underlying GPC is important for the improvement of rice grain quality, and the main obstacle to date is the absence of key genes/QTLs regulating GPC. Primary mapping leads to a large confident interval and poor repeatability of target QTLs, which makes it difficult to find tightly linked markers for marker-assisted selection. Validation and delimitation of $q G P C 1-1$ contributed to the facilitation of marker-assisted selection in rice breeding for high nutritional quality. Furthermore, based on these results, fine mapping and map-based cloning of $q G P C 1-1$ is under way.

Mapping and isolation of QTLs need a high efficiency method to measure GPC. Cloning of QTLs controlling the natural variation of GPC is the important step toward uncovering the regulatory mechanism underlying this quantitative trait. However, map-based cloning of a QTL needs phenotype and genotype information of a massive number of samples, suggesting a rapid and easy operation method for GPC is necessary. Using the NIRS system, the GPC value can be directly measured once the brown rice is grinded into flour.

Compared with the NIRS, the KND method for GPC in rice needs further lengthy operation, which is time-consuming and laborious. More importantly, high correlation between the GPC values determined by the NIRS and KND methods was observed in the HHZ/JZ1560 RIL population across 
two years. Only two minor QTLs ( $q$ GPC10 and $q G P C 11-1$ ) were detected using the single measurement method of GPC, the remaining twelve QTLs were identical to be identified by both methods in 2016 or 2017 (Figures 2 and 3, Table 3). Comparative analysis between the two methods suggested that NIRS could be a feasible strategy for the mapping and map-based cloning of QTLs for GPC instead of the KND method. In recent years, NIRS has been successfully employed in the isolation and characterization of two major QTLs for GPC, $q P C 1$ and $q G P C-10[6,7]$.

Accompanied with the development of DNA sequence techniques, the sequencing cost decreases continuously and more and more high-density genetic maps have been constructed to detect QTLs for different traits through genotyping-by-sequencing $[21,27,28]$. In the present study, QTLs for GPC were mapped simultaneously using a high-density genetic map with 18,194 SNP markers identified by GBR and a low-density map with 208 gel-based SSR and InDel markers in the same RIL population. Although the same number of QTLs were identified using the different resolution genetic maps, 7 of 14 and 3 of 14 QTLs were repeatedly detected across two years in the high- and low-density genetic maps, respectively. Identification of more stably expressed QTLs might be attributed to the increased detection power resulted by the saturated SNP markers in the high-density genetic map.

\section{Materials and Methods}

\subsection{Plant Materials and Field Experiments}

The HHZ/JZ1560 mapping population consisting of 280 RILs was developed using the single-seed descendent method by Ying et al. [29]. HHZ is an indica variety as the female parent with small grains and being widely cultivated in China, while JZ1560 is a japonica rice accession with very large grains (Figure S2). Three RH-derived $\mathrm{F}_{2}$ populations (WB01, WB02 and WB03) consisting 180, 137 and 115 individuals, respectively, were originated from $3 \mathrm{RH}$ plants that were selected from one line of the $\mathrm{F}_{7}$ HHZ/JZ1560 RIL population. The three populations were used to validate the target QTL, qGPC1-1.

All the populations, together with the two parents, were planted with the spacing of $16.7 \mathrm{~cm}$ between plants and $26.7 \mathrm{~cm}$ between rows during the rice-growing seasons in the experimental field of China National Rice Research Institute, Hangzhou (120.2 ${ }^{\circ}$ E, 30.3 $\left.{ }^{\circ} \mathrm{N}\right)$, China. Eighteen plants per RIL were transplanted and the middle four plants were harvested in bulk at maturity for the measurement of GPC in 2016 and 2017. The three RH-derived populations were tested for one year in 2018. Field management was conducted according to the common practice in rice production. Fertilizer was applied for each cultivation year as follows: $375 \mathrm{~kg} / \mathrm{ha}$ compound fertilizer $\left(\mathrm{N}-\mathrm{P}_{2} \mathrm{O}_{5}-\mathrm{K}_{2} \mathrm{O}: 14: 16: 15\right)$ as the basal fertilizer, $52 \mathrm{~kg} / \mathrm{ha}$ nitrogen at seedling stage, $69 \mathrm{~kg} / \mathrm{ha}$ nitrogen at 5 days after transplanting and $150 \mathrm{~kg} / \mathrm{ha}$ potassium at 20 days after transplanting.

\subsection{GPC Measurement}

GPC of brown rice flour for each RIL was carefully measured by the KND and NIRS methods, respectively. Rice grains were harvested after maturity and stored at room temperature for at least three months before the GPC measurement. The fully filled grains were de-hulled into brown rice by a rice huller (JLGJ-45, Taizhou, Zhejiang, China), then the brown rice was grinded into flour by a grinder (IKA TUBE-MILL, Staufen, Germany). Brown rice flour samples were directly used to determine GPC by NIRS (Foss, Sweden). Each sample was assayed twice and the mean values were used for further analysis. GPC was calculated according to the modified NIRS model constructed by Xie et al. [23].

For the KND method to measure GPC, $0.2 \mathrm{~g}$ brown rice flour, $1.0 \mathrm{~g}$ catalyst $\left(\mathrm{CuSO}_{4}: \mathrm{Na}_{2} \mathrm{SO}_{4}=1: 10\right)$ and $4.0 \mathrm{~mL}$ of $\mathrm{H}_{2} \mathrm{SO}_{4}$ were first added into a $100-\mathrm{mL}$ digestion tube in turn. Then the mixture was immediately heated at $420^{\circ} \mathrm{C}$ for $2 \mathrm{~h}$ in a digestion stove. After the solution being digested, the mixture

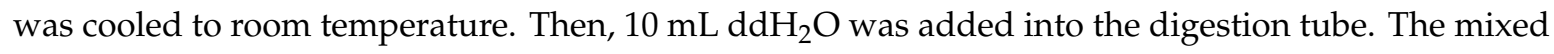
solution was analyzed using a Kjeltec 8400 Autoanalyzer (Foss, Sweden). GPC of the rice flour was calculated from the total $\mathrm{N}$ content by multiplying a conversion factor of 5.95 [30]. The assay for each sample was conducted with two replicates and the means were used for further analysis. 


\subsection{Genetic Map and DNA Marker Analysis}

To compare the detection power of GPC QTL, high-density and low-density linkage maps of the RIL population were both used to map QTL, respectively. The high-density linkage map constructed by one of the GBR method, specific length amplified fragment, was composed of 18,194 SNP markers and spanned $2132.56 \mathrm{cM}$ with an average genetic distance of $0.12 \mathrm{cM}$. In our previous study, we constructed the SLAF library for each RIL and the products were further sequenced using Illumina HiSeq 2500 system (Illumina, Inc.; San Diego, CA, USA) [29]. Polymorphism loci between the parents were identified for the selection of high-quality SNP markers after filtering out the low-quality raw reads. SNP markers with more than $20 \%$ missing data and the segregation distortion were further filtered out. A total of 18,194 high quality SNP markers were used to genotype the 280 RILs. The low-density linkage map constructed by the gel-based method consisted of 121 SSR and 87 InDel markers and spanned $1399.40 \mathrm{cM}$ with an average distance of $7.61 \mathrm{cM}$.

For the three RH-derived populations, leaf samples of each individual were extracted for genomic DNA through the modified CTAB method [31]. To genotype the three RH-derived populations, a total of six InDel markers (Table S2) in the mapping interval of $q$ GPC1-1 were designed with Primer3.0 (http://primer3.ut.ee/) based on the 30-fold genome resequencing of the parents, HHZ and JZ1560. According to our previous study [32], the PCR was performed in $10-\mu \mathrm{L}$ reactions containing $2 \times$ Taq MasterMix (CW0682, CWBIO) 5- $\mu \mathrm{L}, 0.4 \mu \mathrm{M}$ of each primer and $50 \mathrm{ng}$ DNA template. The PCR program was set as an initial denaturation at $94^{\circ} \mathrm{C}$ for $2 \mathrm{~min}$, then followed by 30 cycles of $30 \mathrm{~s}$ at $94{ }^{\circ} \mathrm{C}, 30 \mathrm{~s}$ at $55^{\circ} \mathrm{C}$ and $30 \mathrm{~s}$ at $72{ }^{\circ} \mathrm{C}$, and finally $2 \mathrm{~min}$ at $72{ }^{\circ} \mathrm{C}$. The PCR products were analyzed on $2.5 \%$ agarose gels.

\subsection{QTL Mapping and Statistical Analysis}

Using the high-density genetic map, QTL analysis for GPC was performed by R/qtl software with the method of composite interval mapping (CIM) [33], taking the two years for the RIL population as two environments. A threshold of LOD $>2.0$ was used for detecting a putative QTL. Using the low-density genetic map, QTLs controlling GPC were identified by Windows QTL Cartographer 2.5 [34]. Identification of QTLs was performed using CIM. The LOD threshold for claiming a QTL was also fixed as a LOD score of 2.0. QTL analysis in the three RH-derived populations was also conducted by CIM using Windows QTL Cartographer 2.5 [34].

Basic descriptive statistics, including mean, standard deviation, coefficient of variation, range, skewness and kurtosis, and correlation analysis for GPC in the RIL population were performed with Microsoft Excel 2016 for Windows. The $t$-test of the two parents was performed with the SAS program [35].

\section{Conclusions}

Based on genotyping-by-resequencing, a total of 14 QTLs controlling GPC were identified with an indica/japonica (HHZ/JZ1560) RIL population in 2016 and 2017. Of the 14 QTLs, 13 QTLs showed similar chromosome regions with the QTLs for GPC documented in previous studies. The $q$ GPC10 with a minor effect was newly detected in this study. Seven of the fourteen QTLs were repeatedly identified across two years. The stably inherited $q G P C 1-1$ with a relatively large effect was validated and delimited to a $\sim 862 \mathrm{~kb}$ region flanked by JD1006 and JD1075 on the short arm of chromosome 1, which is helpful for the construction of a marker-assisted selection system to improve rice grain qualities and further map-based cloning of this QTL. Our results indicated that instead of the KND method with lengthy operation, the NIRS with rapid and easy operation was a feasible strategy for measuring a massive collection of samples in the mapping and map-based cloning of GPC QTL. More stably expressed QTLs identified in the genetic map based on genotyping-by-resequencing suggested that high-density map enhanced the detection power of minor QTLs. 
Supplementary Materials: Supplementary materials can be found at http://www.mdpi.com/1422-0067/21/2/408/s1.

Author Contributions: Conceptualization, X.-J.S.; Data curation, Y.-B.W., G.L. and Y.-C.C.; Formal analysis, Y.-J.Z.; Investigation, J.-Y.Y.; Methodology, H.-Z.C. and J.-Z.Y.; Supervision, J.-Z.Y.; Writing-original draft, Y.-B.W.; Writing-review \& editing, G.L. All authors have read and agreed to the published version of the manuscript.

Funding: This research was funded by Chinese High-yielding Transgenic Program (Grant No. 2016ZX08001-004).

Acknowledgments: We thank L. Xie at China National Rice Research Institute for the measurements of grain protein content through near infrared reflectance spectroscopy.

Conflicts of Interest: The authors declare no conflict of interest.

\section{References}

1. Li, Y.; Fan, C.; Xing, Y.; Yun, P.; Luo, L.; Yan, B.; Peng, B.; Xie, W.; Wang, G.; Li, X.; et al. Chalk5 encodes a vacuolar $\left.\mathrm{H}^{+}\right)$-translocating pyrophosphatase influencing grain chalkiness in rice. Nat. Genet. 2014, 46, 398-404. [CrossRef] [PubMed]

2. Zhao, D.S.; Li, Q.F.; Zhang, C.Q.; Zhang, C.; Yang, Q.Q.; Pan, L.X.; Ren, X.Y.; Lu, J.; Gu, M.H.; Liu, Q.Q. GS9 acts as a transcriptional activator to regulate rice grain shape and appearance quality. Nat. Commun. 2018, 9 , 1240. [CrossRef] [PubMed]

3. Zhong, M.; Wang, L.Q.; Yuan, D.J.; Luo, L.J.; Xu, C.G.; He, Y.Q. Identification of QTL affecting protein and amino acid contents in rice. Rice Sci. 2011, 18, 187-195. [CrossRef]

4. Kashiwagi, T.; Munakata, J. Identification and characteristics of quantitative trait locus for grain protein content, TGP12, in rice (Oryza sativa L.). Euphytica 2018, 214, 165. [CrossRef]

5. Lin, R.; Luo, Y.; Liu, D.; Huang, C. Determination and analysis on principal qualitative characters of rice germplasm. In Rice Germplasm Resources in China; Ying, C., Ed.; Agricultural Science and Technology Publisher of China: Beijing, China, 1993; pp. 83-93.

6. Peng, B.; Kong, H.; Li, Y.; Wang, L.; Zhong, M.; Sun, L.; Gao, G.; Zhang, Q.; Luo, L.; Wang, G.; et al. OsAAP6 functions as an important regulator of grain protein content and nutritional quality in rice. Nat. Commun. 2014, 5, 4847. [CrossRef]

7. Yang, Y.; Guo, M.; Sun, S.; Zou, Y.; Yin, S.; Liu, Y.; Tang, S.; Gu, M.; Yang, Z.; Yan, C. Natural variation of OsGluA2 is involved in grain protein content regulation in rice. Nat. Commun. 2019, 10, 1949. [CrossRef]

8. Tan, Y.F.; Sun, M.; Xing, Y.Z.; Hua, J.P.; Sun, X.L.; Zhang, Q.F.; Corke, H. Mapping quantitative trait loci for milling quality, protein content and color characteristics of rice using a recombinant inbred line population derived from an elite rice hybrid. Theor. Appl. Genet. 2001, 103, 1037-1045. [CrossRef]

9. Wada, T.; Uchimura, Y.; Ogata, T.; Tsubone, M.; Matsue, Y. Mapping of QTLs for physicochemical properties in japonica rice. Breed. Sci. 2006, 56, 253-260. [CrossRef]

10. Zhang, W.; Bi, J.; Chen, L.; Zheng, L.; Ji, S.; Xia, Y.; Xie, K.; Zhao, Z.; Wang, Y.; Liu, L.; et al. QTL mapping for crude protein and protein fraction contents in rice (Oryza sativa L.). J. Cereal Sci. 2008, 48, 539-547. [CrossRef]

11. Kepiro, J.L.; McClung, A.M.; Chen, M.H.; Yeater, K.M.; Fjellstrom, R.G. Mapping QTLs for milling yield and grain characteristics in a tropical japonica long grain cross. J. Cereal Sci. 2008, 48, 477-485. [CrossRef]

12. Yu, Y.H.; Li, G.; Fan, Y.Y.; Zhang, K.Q.; Min, J.; Zhu, Z.W.; Zhuang, J.Y. Genetic relationship between grain yield and the contents of protein and fat in a recombinant inbred population of rice. J. Cereal Sci. 2009, 50, 121-125. [CrossRef]

13. Zheng, L.; Zhang, W.; Chen, X.; Ma, J.; Chen, W.; Zhao, Z.; Zhai, H.; Wan, J. Dynamic QTL analysis of rice protein content and protein index using recombinant inbred lines. J. Plant Biol. 2011, 54, 321-328. [CrossRef]

14. Yoshida, S.; Ikegami, M.; Kuze, J.; Sawada, K.; Hashimoto, Z.; Ishii, T.; Nakamura, C.; Kamijima, O. QTL analysis for plant and grain characters of sake-brewing rice using a doubled haploid population. Breed. Sci. 2002, 52, 309-317. [CrossRef]

15. Aluko, G.; Martinez, C.; Tohme, J.; Castano, C.; Bergman, C.; Oard, J.H. QTL mapping of grain quality traits from the interspecific cross Oryza sativa x O. glaberrima. Theor. Appl. Genet. 2004, 109, 630-639. [CrossRef] [PubMed]

16. Hu, Z.L.; Li, P.; Zhou, M.Q.; Zhang, Z.H.; Wang, L.X.; Zhu, L.H.; Zhu, Y.G. Mapping of quantitative trait loci (QTLs) for rice protein and fat content using doubled haploid lines. Euphytica 2004, 135, 47-54. [CrossRef]

17. Bruno, E.; Choi, Y.S.; Chung, I.K.; Kim, K.M. QTLs and analysis of the candidate gene for amylose, protein, and moisture content in rice (Oryza sativa L.). 3 Biotech 2017, 7, 40. [CrossRef] [PubMed] 
18. Liu, X.; Wan, X.; Ma, X.; Wan, J. Dissecting the genetic basis for the effect of rice chalkiness, amylose content, protein content, and rapid viscosity analyzer profile characteristics on the eating quality of cooked rice using the chromosome segment substitution line population across eight environments. Genome 2011, 54, 64-80.

19. Yang, Y.; Guo, M.; Li, R.; Shen, L.; Wang, W.; Liu, M.; Zhu, Q.; Hu, Z.; He, Q.; Xue, Y.; et al. Identification of quantitative trait loci responsible for rice grain protein content using chromosome segment substitution lines and fine mapping of $q P C-1$ in rice (Oryza sativa L.). Mol. Breed. 2015, 35, 130. [CrossRef]

20. Zheng, L.; Zhang, W.; Liu, S.; Chen, L.; Liu, X.; Chen, X.; Ma, J.; Chen, W.; Zhao, Z.; Jiang, L.; et al. Genetic relationship between grain chalkiness, protein content, and paste viscosity properties in a backcross inbred population of rice. J. Cereal Sci. 2012, 56, 153-160. [CrossRef]

21. Wang, Y.; VandenLangenberg, K.; Wen, C.; Wehner, T.C.; Weng, Y. QTL mapping of downy and powdery mildew resistances in PI 197088 cucumber with genotyping-by-sequencing in RIL population. Theor. Appl. Genet. 2018, 131, 597-611. [CrossRef]

22. Sun, X.; Liu, D.; Zhang, X.; Li, W.; Liu, H.; Hong, W.; Jiang, C.; Guan, N.; Ma, C.; Zeng, H.; et al. SLAF-seq: An efficient method of large-scale de novo SNP discovery and genotyping using high-throughput sequencing. PLoS ONE 2013, 8, e58700. [CrossRef] [PubMed]

23. Xie, L.H.; Tang, S.Q.; Chen, N.; Luo, J.; Jiao, G.A.; Shao, G.N.; Wei, X.J.; Hu, P.S. Optimisation of near-infrared reflectance model in measuring protein and amylose content of rice flour. Food Chem. 2014, 142, 92-100. [CrossRef] [PubMed]

24. Li, J.; Xiao, J.; Grandillo, S.; Jiang, L.; Wan, Y.; Deng, Q.; Yuan, L.; McCouch, S.R. QTL detection for rice grain quality traits using an interspecific backcross population derived from cultivated Asian (O. sativa L.) and African (O. glaberrima S.) rice. Genome 2004, 47, 697-704. [CrossRef] [PubMed]

25. Yun, Y.T.; Chung, C.T.; Lee, Y.J.; Na, H.J.; Lee, J.C.; Lee, S.G.; Lee, K.W.; Yoon, Y.H.; Kang, J.W.; Lee, H.S.; et al. QTL mapping of grain quality traits using introgression lines carrying Oryza rufipogon chromosome segments in japonica rice. Rice (NY) 2016, 9, 62. [CrossRef] [PubMed]

26. International Rice Genome Sequencing Project. The map-based sequence of the rice genome. Nature 2005, 436, 793-800. [CrossRef] [PubMed]

27. Lu, X.; Xiong, Q.; Cheng, T.; Li, Q.T.; Liu, X.L.; Bi, Y.D.; Li, W.; Zhang, W.K.; Ma, B.; Lai, Y.C.; et al. A PP2C-1 allele underlying a quantitative trait locus enhances soybean 100-seed weight. Mol. Plant 2017, 10, 670-684. [CrossRef] [PubMed]

28. Zhu, Z.; Li, X.; Wei, Y.; Guo, S.; Sha, A. Identification of a novel QTL for panicle length from wild rice (Oryza minuta) by specific locus amplified fragment sequencing and high density genetic mapping. Front. Plant Sci. 2018, 9, 1492. [CrossRef]

29. Ying, J.Z.; Ma, M.; Bai, C.; Huang, X.H.; Liu, J.L.; Fan, Y.Y.; Song, X.J. TGW3, a major QTL that negatively modulates grain length and weight in rice. Mol. Plant 2018, 11, 750-753. [CrossRef]

30. Sotelo, A.; Sousa, V.; Montalvo, I.; Hernandez, M.; Hernandez-Arago, L. Chemical composition of different fractions of 12 Mexican varieties of rice obta. Cereal Chem. 1990, 67, 209-212.

31. Murray, M.G.; Thompson, W.F. Rapid isolation of high molecular weight plant DNA. Nucleic Acids Res. 1980, 8, 4321-4325. [CrossRef]

32. Zhang, Z.H.; Zhu, Y.J.; Wang, S.L.; Fan, Y.Y.; Zhuang, J.Y. Importance of the interaction between heading date genes Hd1 and Ghd7 for controlling yield traits in rice. Int. J. Mol. Sci. 2019, 20, 516. [CrossRef] [PubMed]

33. Arends, D.; Prins, P.; Jansen, R.C.; Broman, K.W. R/qtl: High-throughput multiple QTL mapping. Bioinformatics 2010, 26, 2990-2992. [CrossRef] [PubMed]

34. Wang, S.; Basten, C.J.; Zeng, Z.B. Windows QTL Cartographer 2.5; Department of Statistics, North Carolina State University: Raleigh, NC, USA, 2006.

35. SAS Institute Inc. SAS/STAT User's Guide; SAS Institute: Cary, NC, USA, 1999.

(C) 2020 by the authors. Licensee MDPI, Basel, Switzerland. This article is an open access article distributed under the terms and conditions of the Creative Commons Attribution (CC BY) license (http://creativecommons.org/licenses/by/4.0/). 